\title{
MicroRNA-103a regulates the calcification of vascular smooth muscle cells by targeting runt-related transcription factor 2 in high phosphorus conditions
}

\author{
LEI HE, JINSHENG XU, YALING BAI, HUIRAN ZHANG, WEI ZHOU, MEIJUAN CHENG, \\ DONGXUE ZHANG, LU ZHANG and SHENGLEI ZHANG
}

\begin{abstract}
Department of Nephrology, The Fourth Hospital of Hebei Medical University, Hebei Key Laboratory of Vascular Calcification in Kidney Disease, Hebei Clinical Research Center for Chronic Kidney Disease, Shijiazhuang, Hebei 050011, P.R. China
\end{abstract}

Received October 15, 2019; Accepted May 24, 2021

DOI: $10.3892 / \mathrm{etm} .2021 .10468$

\begin{abstract}
Vascular calcification, such as atherosclerosis, is a serious complication of chronic kidney disease that is characterized by tunica media calcification, and has gained increasing attention from researchers. The commonly observed association between vascular calcification and osteoporosis suggests a link between bone and vascular disorders. As microRNAs (miRNAs) have a wide range of gene regulation functions, such as cell proliferation, apoptosis, stress and transdifferentiation, the current study aimed to determine whether miRNAs play a vital role in the calcification and osteoblastic differentiation of rat thoracic aorta vascular smooth muscle cells (VSMCs). Gene expression analysis was performed on seven miRNAs (miR-29a, -30b, -103a, -125b, -133a, -143 and -211) that maybe potentially involved in the differentiation of smooth muscle cells into osteoblastic cells. The results showed that the levels of miR-29a, $-30 b,-103 a,-125 b$ and -143 were markedly reduced in the VSMC calcification model, particularly miR-103a, whereas runt-related transcription factor 2 (RUNX2) expression was increased. Furthermore, it was found that the expression of $R U N X 2$ was significantly decreased following the upregulation of miR-103a, and that the expression of $R U N X 2$ was significantly increased by downregulating miR-103a in VSMCs. Therefore, it was concluded that miR-103a plays a notable role in the transdifferentiation of the VSMCs in high phosphorus-induced calcification by targeting
\end{abstract}

Correspondence to: Professor Jinsheng $\mathrm{Xu}$, Department of Nephrology, The Fourth Hospital of Hebei Medical University, Hebei Key Laboratory of Vascular Calcification in Kidney Disease, Hebei Clinical Research Center for Chronic Kidney Disease, 12 Jiankang Road, Shijiazhuang, Hebei 050011, P.R. China E-mail: xjs5766@126.com

Key words: vascular smooth muscle cells, $\beta$-glycerophosphate, microRNAs, calcification, runt-related transcription factor 2 , chronic kidney disease the regulation of $R U N X 2$, and may therefore constitute a new target for the diagnosis and treatment of vascular calcification.

\section{Introduction}

Cardiovascular disease is the primary complication and leading cause of death in patients with chronic kidney disease (CKD) (1). A potentially life-threatening cardiovascular factor in patients with CKD is the presence of vascular calcification, which exists in up to $47-83 \%$ of patients with CKD (2-4). Vascular calcification in patients with CKD can be broadly divided into two types: Intimal calcification and medial calcification, with the latter observed more commonly (5). A previous study has suggested that the mechanism of vascular calcification is a cell-mediated, highly regulated process similar to osteogenesis (6). During this process, vascular smooth muscle cells (VSMCs) undergo a series of pathological changes that eventually result in the generation of osteoblast-like cells (phenotypic transition) (7).

MicroRNAs (miRNAs/miRs) are a class of small, single-stranded, non-coding RNAs that modulate gene expression by binding to the $3^{\prime}$ untranslated region ( $\left.3^{\prime} \mathrm{UTR}\right)$ of their target genes, causing translational suppression and/or mRNA degradation $(8,9)$. Accumulating evidence indicates that miRNAs play notable roles in a wide range of biological processes, including cellular proliferation, apoptosis and differentiation; therefore, they are involved in a variety of pathologies, most notable cancer (10). Several miRNAs have been identified to negatively regulate osteoblast differentiation by inhibiting mineralization via silencing of the key transcription factors of osteogenesis, such as runt-related transcription factor 2 (Runx2), osteopontin (OPN), osterix or distal-less homeobox 5 (11).

The osteogenesis transcription factor Runx 2 , as an essential transcription factor in osteoblast differentiation and chondrocyte maturation, and plays a notable role in the calcification of VSMCs. Under normal conditions, Runx2 is expressed at low levels, but is markedly increased in calcified atherosclerotic plaques, suggesting that Runx 2 may be a significant factor involved in vascular calcification. A previous study revealed 
that multiple miRNAs promote osteogenesis through direct or indirect regulation of Runx2 expression. Zhang et al (12) reported that miRNA-133a-5p inhibits the expression of osteoblast differentiation-associated markers by targeting the $3^{\prime}$ UTR of RUNX2. miR-29a and miR-125b indirectly targeting Runx2 via its co-repressors or co-activators may lead to increased or decreased regulation of osteoblast differentiation, respectively (13).

Notably, the miRBase database platform suggested the sequence-specific base pairing of the 'seed region' at the 5' or 3 ' ends of miR-30b, $-103 a,-133 a,-143$ and -211 with the 3'-UTR binding sites of Runx2, which regulated its expression. Therefore, miR-29a, -30b, -103a, -125b, -133a, -143 and -211 were selected to confirm whether miRNAs were involved in the regulation of vascular calcification under high phosphorus conditions, and if so, the underlying mechanisms by which it functions in this context.

\section{Materials and methods}

Ethics statement. All experimental programs were reviewed and approved by The Animal Care and Use Institutional Committee of Hebei Medical University (approval no. 2020ky189; Shijiazhuang, China).

Cell culture. A total of 20 adult male Sprague Dawley rats (age, 8 weeks; weight, 200-250 g) were purchased from the Hebei Medical University Lab Animal Center (Shijiazhuang, China) and allowed free access to standard rat chow and tap water, in $22 \pm 1{ }^{\circ} \mathrm{C}$ temperature- and $40-60 \%$ humidity-controlled conditions, with a $12 \mathrm{~h}$ dark/light cycle. The 20 rats were anesthetized with $400 \mathrm{mg} / \mathrm{kg}$ chloral hydrate and intraperitoneally euthanized with $300 \mathrm{mg} / \mathrm{kg}$ sodium pentobarbital (three times the anesthetic dose). Rats were considered dead after breathing had stopped, cardiac arrest was confirmed, righting reflex disappeared and the pupils were dilated. The cause of death for all rats was cardiac arrest. The primary VSMCs were extracted from the thoracic aorta using a previously described method (14). The experiment was usually completed within $30 \mathrm{~min}$ and no peritonitis was observed.

The cells were cultured with DMEM (Gibco; Thermo Fisher Scientific Inc.) containing $4.5 \mathrm{~g} / 1$ glucose supplemented with $15 \% \mathrm{FBS}$, at $37^{\circ} \mathrm{C}\left(5 \% \mathrm{CO}_{2}\right)$ in a humidified atmosphere. To induce calcification, the VSMCs were seeded at a density of 5,000 cells $/ \mathrm{cm}^{2}$ in six-well dishes and cultured in $10 \mathrm{mmol} / 1$ $\beta$-glycerophosphate $(\beta-\mathrm{GP}), 10 \mathrm{~mol} / \mathrm{l}$ insulin and $50 \mathrm{mg} / \mathrm{ml}$ ascorbic acid in the presence of $15 \%$ FBS (HyClone; Cytiva), and the control groups were cultured in identical conditions but without the $\beta$-GP. The culture medium was replaced every day.

Transfection. The miRNAs were obtained from Guangzhou RiboBio Co., Ltd., and the target sequences were as follows: Mimic miR-103, AGCAGCAUUGUACAGGGCUAUGA; inhibitor miR-103, UCAUAGCCCUGUACAAUGCUGCU; mimic negative control (NC), UUUGUACUACACAAAAGU ACUG; and inhibitor NC, CAGUACUUUUGUGUAGUACAA A. The mimic NC and inhibitor NC were both non-targeting. The miRNA (mimic-103a, inhibitor-103a and control miRNAs) transfection was performed using Lipofectamine ${ }^{\circledR} 2000$ transfection reagent according to the manufacturer's instructions (Invitrogen; Thermo Fisher Scientific, Inc.). Briefly, $6 \mu \mathrm{g}$ miRNA and $10 \mu 1$ Lipofectamine ${ }^{\circledR} 2000$ were mixed for 20 min at room temperature, and subsequently added to the VSMCs cultured in six-well plates. At $72 \mathrm{~h}$ post-transfection, the intracellular RNA and protein were extracted to detect miR-103 and Runx 2 expression by reverse transcription-quantified PCR (RT-qPCR) and western blotting, respectively.

Target miRNA forecast of RUNX2. miRBase (http://www. mirbase.org/) online analysis tool was used to predict the miRNAs targeting Runx2 (15). miRBase was developed by Griffiths-Jones et al (15) to provide users with a more comprehensive relationship between miRNA and target genes (15).

RNA sequencing. VSMC cells were treated with normal or $10 \mathrm{mmol} / \mathrm{l} \beta$-GP medium, followed by the extraction of total RNA by using TRIzol ${ }^{\circledR}$ reagent (Invitrogen; Thermo Fisher Scientific Inc.). The quality of RNA was typically measured by using a NanoDrop spectrophotometer (ND-1000; NanoDrop Technologies; Thermo Fisher Scientific Inc.) and the integrity was assessed by using gel electrophoresis to analysis the ratios of $28 \mathrm{~S}$ to $18 \mathrm{~S}$ ribosomal bands. Then, the RNA was used to prepare the miRNA sequencing library, which included the following steps: 3'-adaptor ligation, 5'-adaptor ligation, cDNA synthesis, PCR amplification and size selection of $\sim 135$ to 155 bp PCR amplified fragments (corresponding to $\sim 15$ to 35 nt small RNAs). The Invitrogen ${ }^{\mathrm{TM}}$ Collibri $^{\mathrm{TM}}$ kit (Invitrogen; Thermo Fisher Scientific Inc.). was used to quantify the sequencing library and $20 \mathrm{pM}$ products were subjected to deep sequencing using an Illumina-Solexa $1 \mathrm{G}$ Genetic Analyzer (Illumina, Inc.). The pre-miRNA databases (known premiRNA from miRBase v.21 plus the newly predicted pre-miRNAs) and Novoalign software (http://www.novocraft. com/products/novoalign/.v.2.07.11; Novocraft Technologies) was used to analysis the data.

Calcification assays. After being cultured with $\beta$-GP at $37^{\circ} \mathrm{C}$ for 14 days, the transfected VSMC cells were washed three times with PBS. The calcium content was determined by spectrophotometry using a calcium assay kit for $24 \mathrm{~h}$ at $4^{\circ} \mathrm{C}$ (BioSino Biotechnology \& Science, Inc.), and then by histology using a $1 \%$ Alizarin red stain at $4^{\circ} \mathrm{C}$ for $24 \mathrm{~h}$. The specific procedures used were described previously (14). The results were then normalized by protein content, which was quantified using a BCA protein assay kit (Beijing Solarbio Science \& Technology Co., Ltd.).

Alkaline phosphatase (ALP) activity. To determine the ALP activity in $\beta$-GP stimulated or miRNA transfected VSMC cells, a phosphorylated nitrophenyl substrate was performed using an alkaline phosphatase assay kit (Nanjing Jiancheng Bioengineering Institute), according to the manufacturer's instructions. The results were calculated according to the curves generated from the standard samples, and normalized by protein concentration.

$R T$ - $q P C R$. Total RNA was extracted from VSMCs using TRIzol ${ }^{\circledR}$ reagent (Invitrogen; Thermo Fisher Scientific, Inc.) according to the manufacturer's protocol and cDNA was 
Table I. Primer pairs used for reverse transcription-quantitative PCR.

Primer

Sequence, 5'-3'

miR-29a-3p forward

miR-29a-3p reverse

miR-30b-5p forward

miR-30b-5p reverse

miR-103a-3p forward

miR-103a-3p reverse

miR-125b-5p forward

miR-125b-5p reverse

miR-133a-3p forward

miR-133a-3p reverse

miR-143-3p forward

miR-143-3p reverse

miR-211-3p forward

miR-211-3p reverse

$5 \mathrm{~S}$ forward

$5 \mathrm{~S}$ reverse

Runx2 forward

Runx2 reverse

GAPDH forward

GAPDH reverse

\author{
AGGAGGTAGCACCATCTGAAATC \\ GTCGTATCCAGTGCAGGGTCCGAGGTATTCGCACTGGATACGACTAACCG \\ TCCTCCTGTAAACATCCTACACT \\ GTCGTATCCAGTGCAGGGTCCGAGGTATTCGCACTGGATACGACAGCTGA \\ AGAGGTTTGGTCCCCTTCAAC \\ GTCGTATCCAGTGCAGGGTCCGAGGTATTCGCACTGGATACGACCAGCTG \\ GCTCCTCCCTGAGACCCTAAC \\ GTCGTATCCAGTGCAGGGTCCGAGGTATTCGCACTGGATACGACTCACAA \\ AGAGGTTTGGTCCCCTTCAAC \\ GTCGTATCCAGTGCAGGGTCCGAGGTATTCGCACTGGATACGACCAGCTG \\ AGGAGGTGAGATGAAGCACTGT \\ GTCGTATCCAGTGCAGGGTCCGAGGTATTCGCACTGGATACGACGAGCTA \\ GGTGTTCCCTTTGTCATCCTT \\ GTCGTATCCAGTGCAGGGTCCGAGGTATTCGCACTGGATACGACAGGCGA \\ CTGGTTAGTACTTGGACGGGAGAC \\ GTCGTATCCAGTGCAGGGTCCGAGGTATTCGCACTGGATACGACCAGGCG \\ CCAGCAGCACTCCATATCTC \\ CAGCGTCAACACCATCATTC \\ GGAGCGAGATCCCTCCAAAAT \\ GGCTGTTGTCATACTTCTCATGG
}

Runx2, runt-related transcription factor 2; miR-, microRNA.

synthesized using superscript reverse transcriptase (Clontech Laboratories, Inc.) at $37^{\circ} \mathrm{C}$ for $15 \mathrm{~min}$ and $85^{\circ} \mathrm{C}$ for $5 \mathrm{sec}$. To investigate the expression of Runx 2 mRNA, qPCR was performed using DyNAmo ColorFlash SYBR Green qPCR kit (Thermo Fisher Scientific, Inc.) according to the following protocol: $40 \mathrm{Cycles}$ of $95^{\circ} \mathrm{C}$ for $5 \mathrm{~min}, 95^{\circ} \mathrm{C}$ for $10 \mathrm{sec}, 55^{\circ} \mathrm{C}$ for $30 \mathrm{sec}$ and $72^{\circ} \mathrm{C}$ for $10 \mathrm{sec}$. The mature miR expression levels were also analyzed from total RNA using the miScript SYBR Green PCR kit (Thermo Fisher Scientific, Inc.) for miR-103a, miR-29a, miR-133a, miR-30b, miR-143, miR-125b and miR-211, according to the manufacturer's instructions. The primer sequences for rat Runx2, GAPDH and the candidate miRNAs are presented in Table I. The expression of the selected miRNAs relative to $5 \mathrm{~s}$, and of Runx 2 relative to GAPDH. The data were analyzed using the Quantstudio ${ }^{\mathrm{TM}} \mathrm{Dx}$ v1.0 software (Thermo Fisher Scientific, Inc.).

Western blotting. Protein was extracted from VSMCs using a lysis buffer containing $150 \mathrm{mmol} / \mathrm{l} \mathrm{NaCl}, 50 \mathrm{mmol} / \mathrm{l}$ Tris (pH 7.4), $1 \%$ Triton X-100, 0.1\% SDS, 1 mmol/l EDTA, $0.5 \%$ sodium deoxycholate, $2 \mathrm{mmol} / 1$ sodium pyrophosphate, $1 \mathrm{mmol} / \mathrm{k} \mathrm{Na}_{3} \mathrm{VO}_{4}, 0.5 \mu \mathrm{g} / \mathrm{ml}$ leupeptin and $0.1 \mathrm{mmol} / 1 \mathrm{PMSF}$. The protein concentration was determined using the BCA method (Sigma-Aldrich; Merck KGaA), and $40 \mu \mathrm{g}$ protein per lane was loaded onto $10 \%$ SDS-PAGE gels for electrophoresis. The specific procedures for electrophoresis and electrotransfer to polyvinylidene difluoride membranes were performed as previously described (14). The blots were incubated with 5\% BSA (Beijing Solarbio Science \& Technology Co., Ltd.) at $37^{\circ} \mathrm{C}$ for $1 \mathrm{~h}$, followed by incubation with specific primary antibodies against Runx2 (1:100 dilution; cat. no. ab23981; Abcam) and GAPDH (1:10,000 dilution; cat. no. AP0063; Bioworld Technology, Inc.) overnight at $4^{\circ} \mathrm{C}$. After rinsing with $1 \%$ Tween-Tris buffered saline, the blots were incubated with peroxidase-conjugated secondary antibody (1:4,000 dilution; cat. no. TA130016; Origene Technologies Inc.) at room temperature for $2 \mathrm{~h}$. Immunodetection was performed using an Enhanced Chemiluminescence kit (Amersham; Cytiva). The expression level of stained proteins quantified by digital image analysis, according to the integrated optical density (IOD) of the positive region. The integrated optical density ratio of the indicated Runx 2 and GAPDH bands was measured by using the Image Pro-Plus v.5.0 software (Media Cybernetics, Inc.) to calculate the relative expression level.

Luciferase assay. pMIR vector of wild-type (WT) RUNX2 3'-UTR and mutant of the RUNX2 3'-UTR were purchased from Guangzhou RiboBio Co., Ltd. The sequences of mimic-103a and mimic negative control (NC) obtained from Guangzhou RiboBio Co., Ltd. were as follows: 5'-AGCAGC AUUGUACAGGGCUAUGA-3' and 5'-UUCUCCGAACGU GUCACGUTT-3', respectively. VSMC cells ( $3 \times 10^{4}$ cells/well) were co-transfected with $200 \mathrm{ng}$ of the mimic-103a and $50 \mathrm{ng}$ of the Wild-type or mutant luciferase plasmid by using Lipofectamine ${ }^{\circledR} 2000$ transfection reagent (Invitrogen; Thermo Fisher Scientific, Inc.). The luciferase assays were performed $48 \mathrm{~h}$ later with the dual-luciferase reporter assay system (Promega Corporation) and FLUOstar photometer (BMG Labtech $\mathrm{GmbH}$ ). Relative luciferase activity of each group was expressed as a percentage of Renilla luciferase 


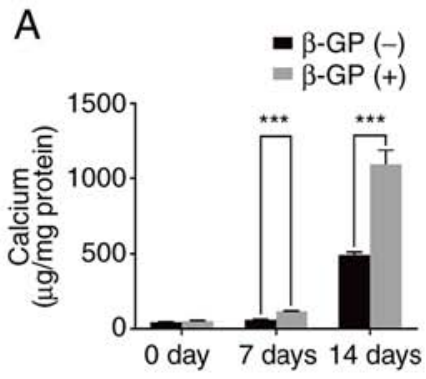

B
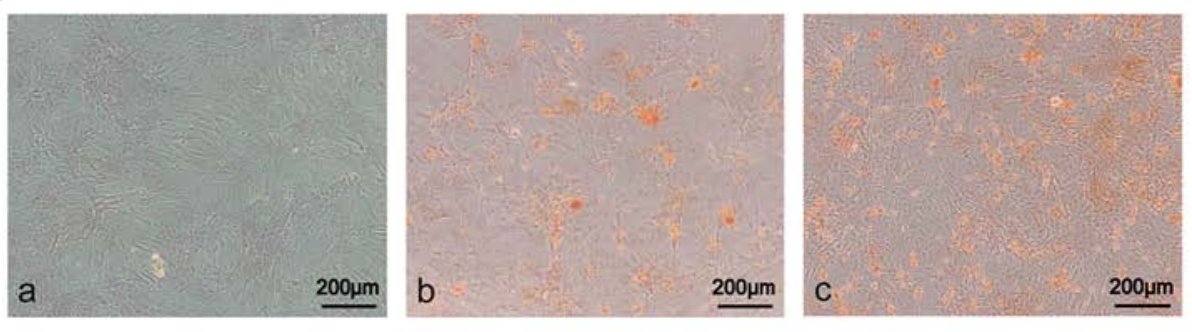

E
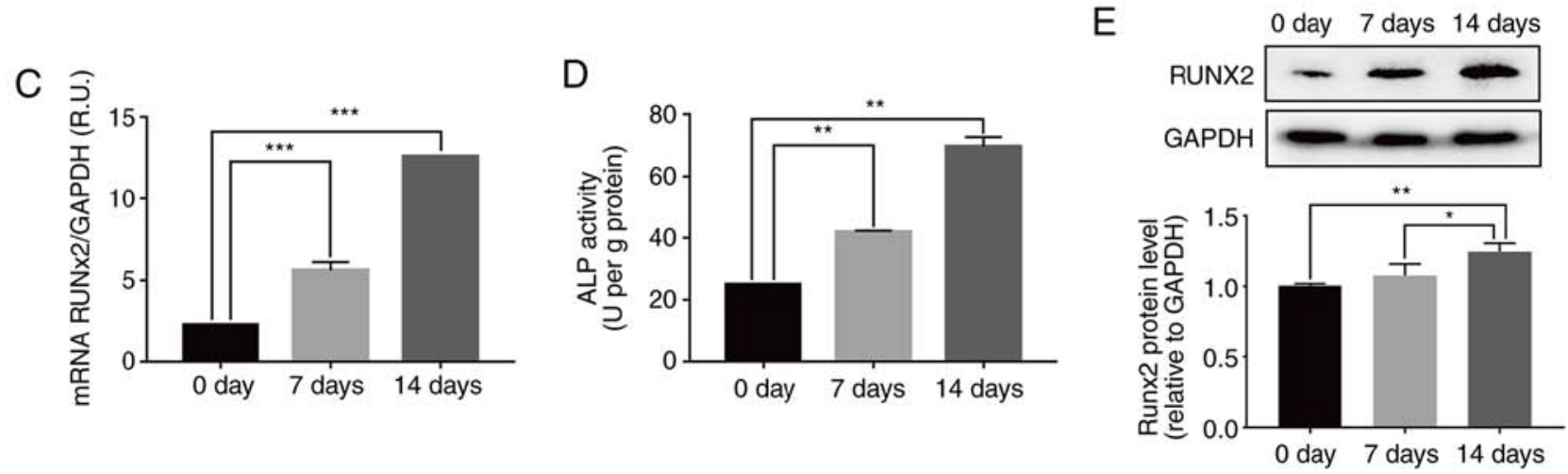

Figure 1. Mineral deposition and calcium content during calcification of VSMCs. (A) Calcium content was quantified by spectrophotometry. (B) Rat thoracic VSMCs treated with $10 \mathrm{mM} \beta$-GP for (a) 0, (b) 7 and (c) 14 days, and subjected to Alizarin Red staining; representative microscopic views are shown; $\mathrm{n}=5$. (C) Runx2 expression and (D) ALP activity during the calcification of VSMCs by radioimmunoassay. (E) Examination of Runx2 expression by reverse transcription-quantitative PCR and western blotting analysis; ${ }^{*} \mathrm{P}<0.05,{ }^{* *} \mathrm{P}<0.01$ and ${ }^{* * *} \mathrm{P}<0.001$. VSMCs, vascular smooth muscle cells; Runx2, runt-related transcription factor 2; ALP, alkaline phosphatase; $\beta$-GP, $\beta$-glycerophosphate.

activity normalized to the corresponding firefly luciferase activity. The relative luciferase activity (\%) represented the expression level of $R U N X 2$.

Statistical analysis. SPSS v17.0 software (SPSS, Inc.) was used for analysis. All experiments were repeated 3 times. The data are presented as the mean \pm standard deviation (unless otherwise stated). For experiments with 2 groups, statistical analyses were performed using Student's unpaired t-test. For experiments with $>2$ groups, the statistical analyses were performed using one-way ANOVA. Furthermore, the Student-Newman-Keuls post hoc test was used to determine the statistical significance of the differences among groups. $\mathrm{P}<0.05$ was considered to indicate a statistically significant difference.

\section{Results}

High phosphorus promotes calcification by inducing the transdifferentiation of VSMCs into osteoblast-like cells. The calcification of VSMCs cultured in medium with $10 \mathrm{mM}$ $\beta$-GP for 0,7 and 14 days was examined. It was found that treatment with high phosphorus levels significantly increased the calcium content of VSMCs in a time-dependent manner, as demonstrated by the calcium content assay (Fig. 1A). Consistent with the changing trend of the calcium content results, VSMCs showed a significant increase in mineralization after 7 days of culture, which was measured by Alizarin red staining, reaching the highest calcification level at day 14 (Fig. 2B). These data suggested that high phosphorus conditions induce calcification in the VSMCs.
To investigate the underlying mechanisms of VSMC calcification induced by high phosphorus, the ALP activity and the mRNA and protein expression of Runx 2 were determined. The results showed that high phosphorus significantly upregulated the ALP activity and mRNA and protein expression levels of Runx2. The level of Runx2 mRNA was significantly increased by 56.9 and $81.48 \%$ after 7 and 14 days of high phosphorus treatment, respectively, compared with the normal control group (Fig. 1C). Furthermore, compared with the normal control group, the protein levels of Runx2 in VSMCs treated with high phosphorus for 7 and 14 days increased significantly, by 19.1 and $25.9 \%$, respectively (Fig. 1E). The ALP activity was significantly increased in a time-dependent manner, similar to the expression of Runx2, in VSMCs treated with high phosphorus (Fig. 1D). These data suggested that the transdifferentiation of VSMCs to osteoblast-like cells may play a prominent role in the high phosphorus-induced calcification of VSMCs.

Identification of differentially expressed miRNAs during the calcification of the VSMCs in high phosphorus conditions. To further investigate the modulation mechanism of high phosphorus in the osteoblast-like cell transdifferentiation of the VSMCs, the expression levels of miRNAs were explored using the RNA sequencing method. The results, presented in Fig. 2A, show that miR-30, miR-125 and miR-143 expression levels decreased, whereas miR-211 expression increased in high phosphorus-treated VSMCs, compared with normal cells.

Furthermore, VSMCs were treated with $10 \mathrm{mmol} / 1 \beta$-GP for 0,7 and 14 days, and the miRNA was extracted for RT-qPCR analysis. The results of gene expression analysis showed 


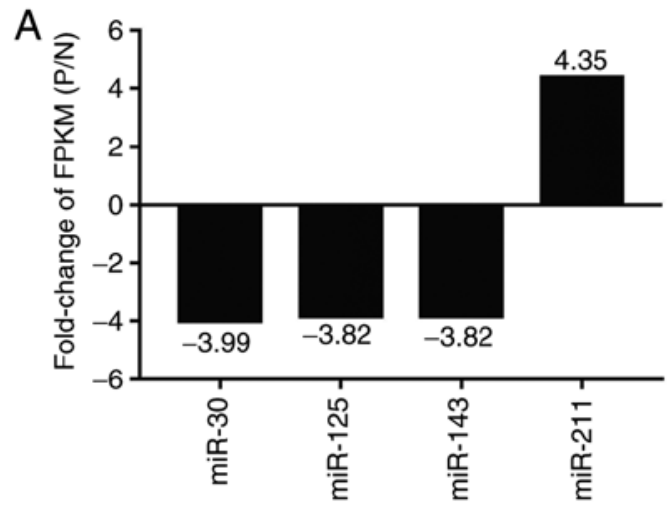

B

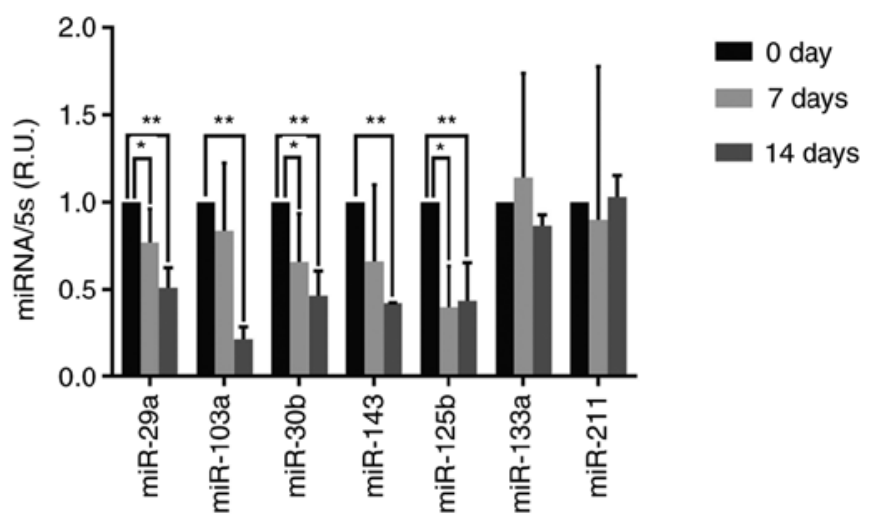

Figure 2. miRNAs regulate $\beta$-GP-induced osteoblastic differentiation of VSMCs. (A) RNA sequencing of mRNAs in VSMCs treated with normal medium and high-phosphorus medium. (B) VSMCs were cultured for 0,7 and 14 days in $10 \mathrm{mM} \beta$-GP. Each miRNA was assessed by reverse transcription-quantitative $\mathrm{PCR} ; \mathrm{n}=5$. ${ }^{*} \mathrm{P}<0.05$ and ${ }^{* *} \mathrm{P}<0.01$. miRNA/miR-, microRNA; VSMCs, vascular smooth muscle cells; $\beta$-GP, $\beta$-glycerophosphate; FPKM, fragments per kilobase of transcript per million mapped; P/N, P, $\beta$-glycerophosphate medium; $N$, normal medium; R.U., relative fluorescence units.

that the expression levels of miR-29a, miR-30b, miR-103a, miR-125b and miR-143 significantly decreased in VSMCs cultured with high phosphorus for 7 days, compared with those in the normal control group; however, the expression of miR-133a and miR-211 did not decrease significantly (Fig. 2B). Moreover, the expression of miR-29a, miR-30b, miR-103a, miR-125b, miR-143, miR-133a and miR-211 was downregulated by $48.85,60.56,53.44,58.11,56.78,36.13$ and $31.24 \%$ in VSMCs cultured with high phosphorus for 14 days, compared with the normal control group, respectively (Fig. 2B). It can be observed that the expression of miR-103a decreased the most in high phosphorus-induced VSMCs, and it was therefore hypothesized that miR-103a was likely to be involved in regulating Runx 2 expression in the high phosphorus-induced VSMCs.

miR-103a inhibits Runx2 expression in the high phosphorusinduced calcification of VSMCs. Considering that the expression of miR-103a showed the most significant reduction out of the selected miRNAs in the VSMC calcification model (at 14 days), miR-103a was selected as the focus of further study. To determine whether miR-103a is involved in the regulation of Runx2 in VSMCs, the mRNA and protein levels of Runx2 were assessed by overexpressing miR-103a (mimic-103a), or reducing miR-103a expression (inhibitor-103a) in normal cultured VSMCs. The results showed a 155-fold increase in the mimic-103a, compared with the mimic-NC group (Fig. 3); and the mRNA and protein levels of Runx 2 decreased by 52.7 and $28.8 \%$ compared with the mimic-NC, respectively, at 14 days post-transfection with mimic-103a (Fig. 4A and B).

Furthermore, inhibitor-103a transfection resulted in a significant decrease in VSMC miR-103a expression, and the expression levels of Runx $2 \mathrm{mRNA}$ and protein were increased by 68.3 and $16.9 \%$, respectively (Fig. 4A and B).

Taken together, these results demonstrated that miR-103a plays a notable role in modulating the expression of Runx2 during high phosphorus-induced phenotype transition of VSMCs into osteoblast-like cells.

Overexpression of miR-103a ameliorates calcification caused by high phosphorus-induced transformation of VSMCs into

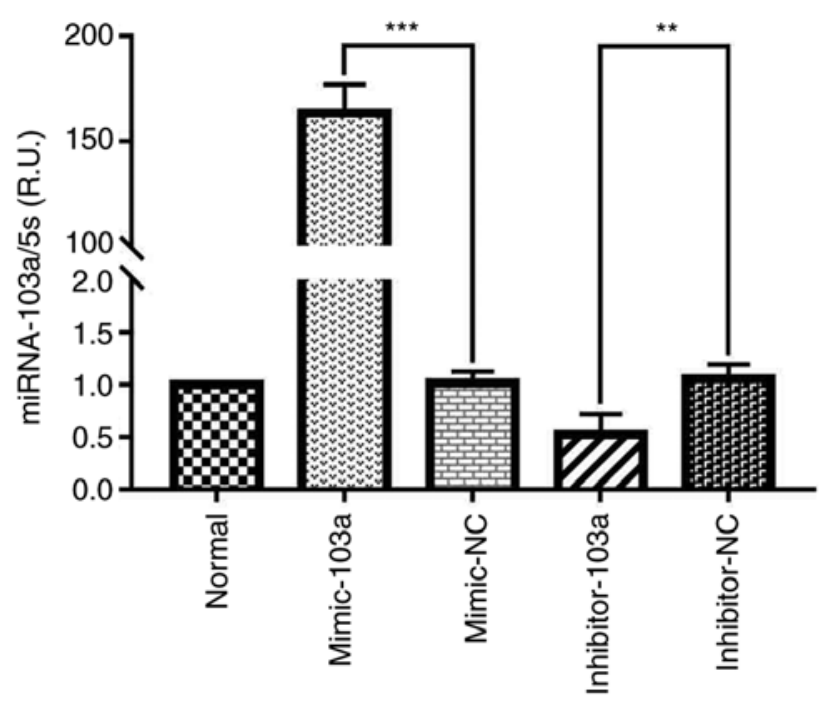

Figure 3. Reverse transcription-quantitative PCR analysis of microRNA-103a levels in vascular smooth muscle cells after treatment with mimic-103a, inhibitor-103a or their NCs (mimic-NC, inhibitor-NC). ${ }^{* *} \mathrm{P}<0.01$ and ${ }^{* * *} \mathrm{P}<0.001$. $\mathrm{NC}$, negative control; miRNA, microRNA; R.U., relative fluorescence units.

osteoblasts. To investigate the effects of miR-103a on cell phenotype transformation, the expression of miR-103 was inhibited in normal cultured VSMCs and examined using Alizarin red staining, as well as calcium content and ALP activity assays. The results of Alizarin red staining revealed that when miR-103a was inhibited, the calcium nodules were increased (Fig. 5A). The results of the calcium content assay demonstrated that following miR-103a-knockdown, calcium content was significantly increased by $x 2.4$ compared with the control group (Fig. 5B). Consistent with the calcium content results, ALP activity assay, indicated a significant increase in ALP activity following miR-103a-knockdown (Fig. 5C). Collectively, these data suggested that the inhibition of miR-103a promoted the transformation of smooth muscle cells into osteoblasts.

To further verify the role of miR-103a, mimic-103a was transfected in VSMCs cultured with high phosphorus and assessed by using Alizarin red staining, calcium content and 
B
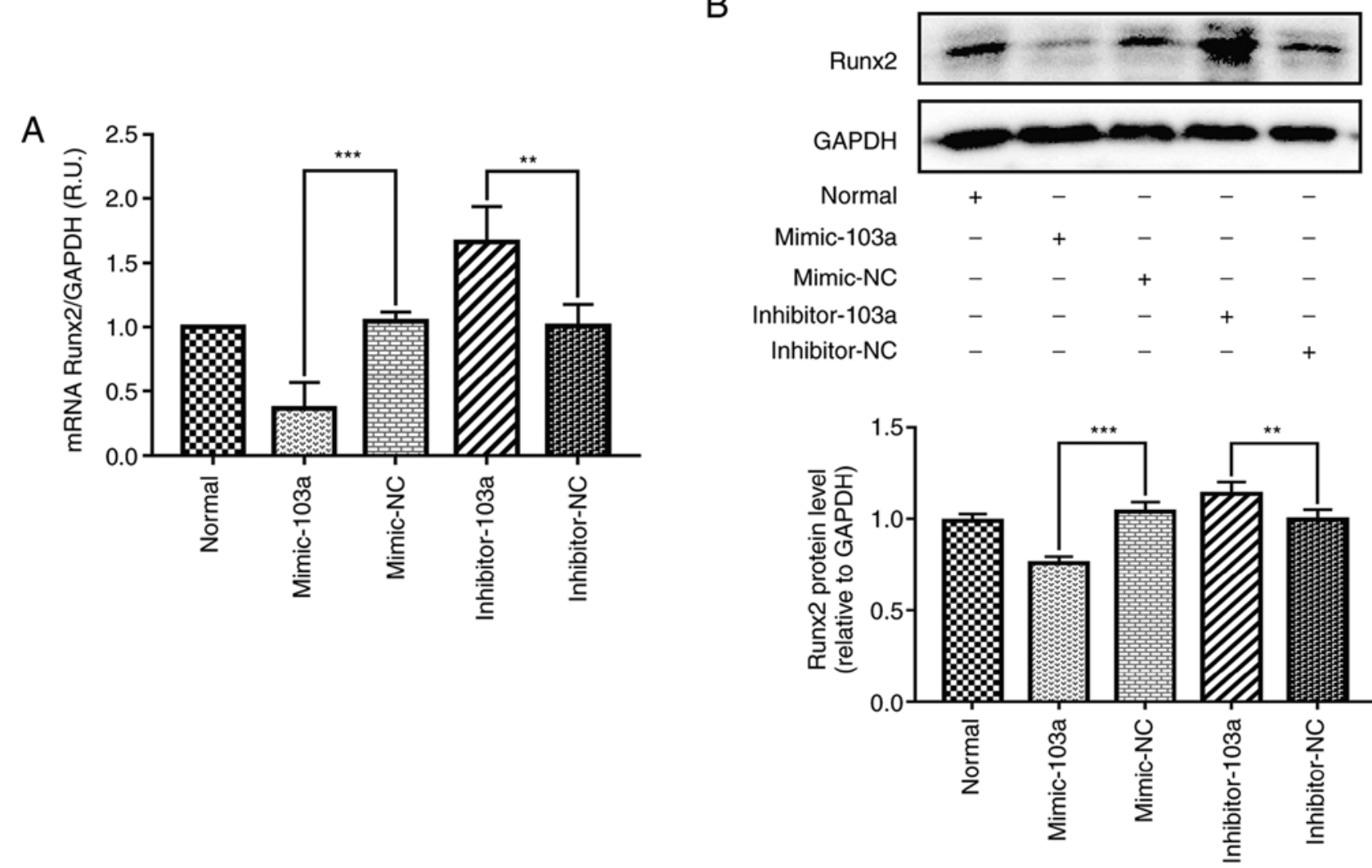

Figure 4. Regulation effects of miRNA-103 on the expression of Runx2. VSMC cells were transfected with miR-103 mimics, or miR-103 inhibitor and the Runx2 expression was observed by RT-qPCR and western blot. (A) RT-q PCR analysis of Runx2 mRNA in vascular smooth muscle cells transfected with mimic-103a or inhibitor-103a. (B) Western blot of Runx2 protein expression in vascular smooth muscle cells transfected with mimic-103a or inhibitor-103a. ${ }^{* *} \mathrm{P}<0.01$ and ${ }^{* * *} \mathrm{P}<0.001$. Runx2, runt-related transcription factor 2; NC, negative control; R.U., relative fluorescence units; RT-q, reverse transcription-quantitative; miR, microRNA.

ALP activity assays. The results of Alizarin red staining showed that overexpression of miR-103a decreased the level of dye incorporation (Fig. 5D) and that the calcium content (Fig. 5E) and ALP activity (Fig. 5F) decreased compared with the control group.

To investigate whether miR-103 directly targeted the 3'-UTR of Runx2, RUNX2 Wild-type or mutant luciferase plasmid and miR-103a mimics were co-transfected into the cells and the relative luciferase activity of each group was then detected. The relative luciferase activity was significantly reduced in cells transfected with the miR-103a mimics and the pLuc-RUNX2 3'-UTR Wild-type plasmid compared with cells transfected with miR-103a natural control and the pLuc-RUNX2 3'-UTR Wild-type plasmid group. However, the relative luciferase activity was not significantly increased between the mutant-RUNX2-transfection and the Wild-type-RUNX2-transfection in the cells with overexpression of the miR-103a (Fig. S1).

Taken together, the aforementioned results indicated that increased expression levels of miR-103a can ameliorate calcification caused by high phosphorus-induced transformation of the VSMCs into osteoblasts.

\section{Discussion}

In the present study, miR-103a was identified as a negative regulator of vascular medial calcification in vitro, potentially by inhibiting the phenotypic transformation of VSMCs into osteoblast-like cells, and downregulating Runx2 expression levels and ALP activity.

Osteoblast-like transformation of VSMCs plays a key role in the pathogenesis of vascular medial calcification (16). It has been reported that VSMCs can acquire osteoblast-like phenotypes and expression of osteogenic markers in the process of vascular calcification (17). The results of the present study showed that the expression of the osteochondrogenic marker Runx2 and the ALP activity were notably increased in calcified VSMCs. Moreover, high phosphorus promoted Runx2 expression and ALP activity in a time-dependent manner. Similarly, a previous study indicated that exposure to elevated phosphorus levels results in the upregulation of Runx2 expression and ALP activity (18). Runx2 is a notable transcription factor in the process of VSMC osteo-/chondrogenic transdifferentiation, which induces the expression of major bone matrix components, including type I collagen (5), osteocalcin (19) and OPN (20). Runx2 can be transcribed from the distal $\mathrm{P} 1$ promoter or the $\mathrm{P} 2$ promoter, resulting in the expression of two different subtypes (Runx2 I and II), which can be expressed in ossified and cartilage cells; both subtypes exhibit similar features in chondrocytes and osteoblasts. However, the mechanisms underlying the osteogenic differentiation of high phosphorus-induced VSMCs are not completely understood and therefore require further research.

As a key regulator of vascular calcification, Runx 2 is likely to be involved in multiple levels of VSMC regulation $(13,21)$. Runx2 has a long 3'-UTR ( 4 kb), presumably containing 
A

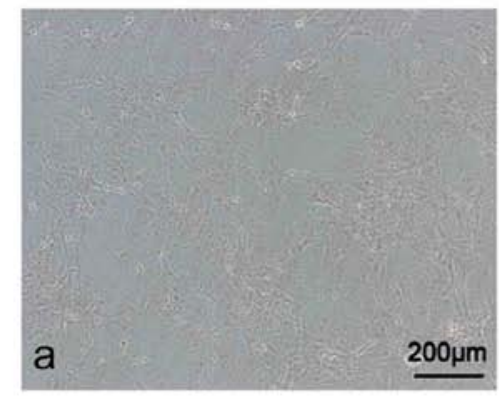

B

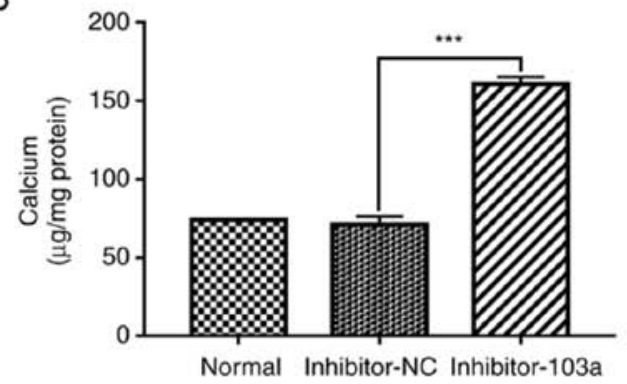

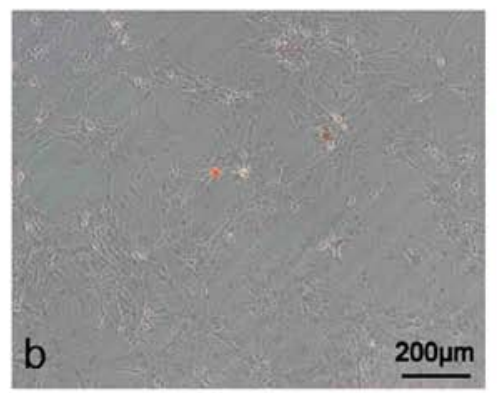

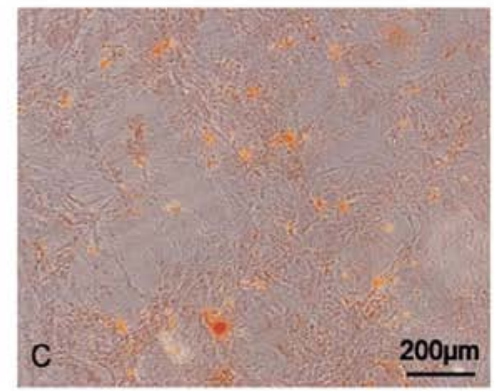

C

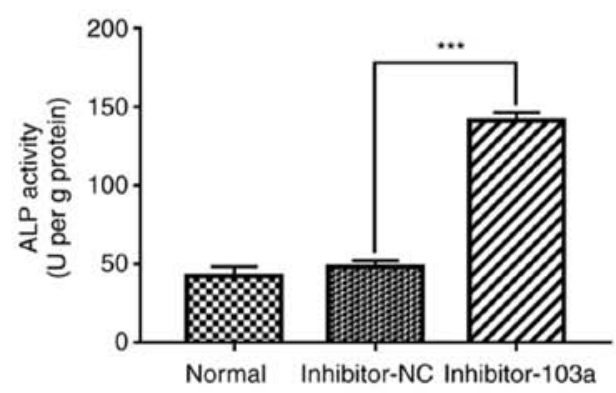

D

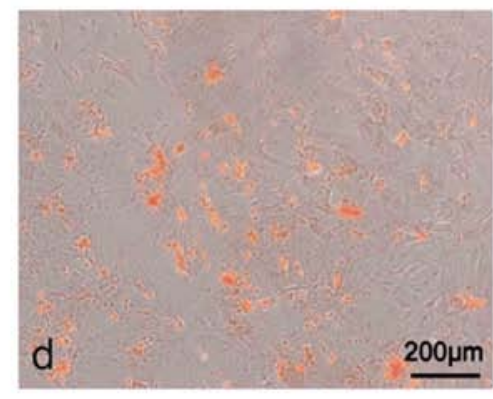

E

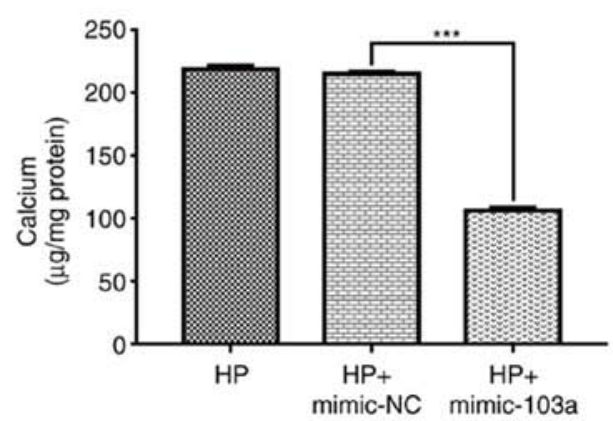

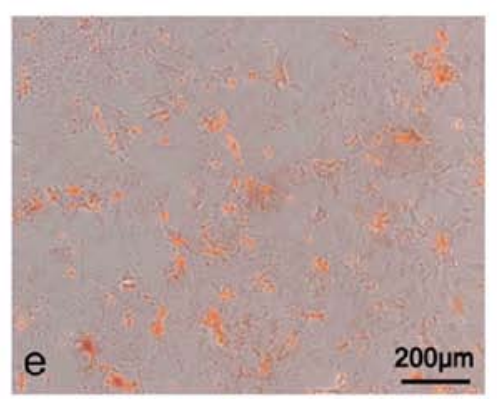

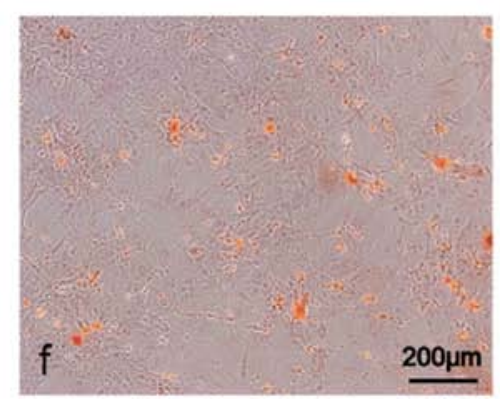

F

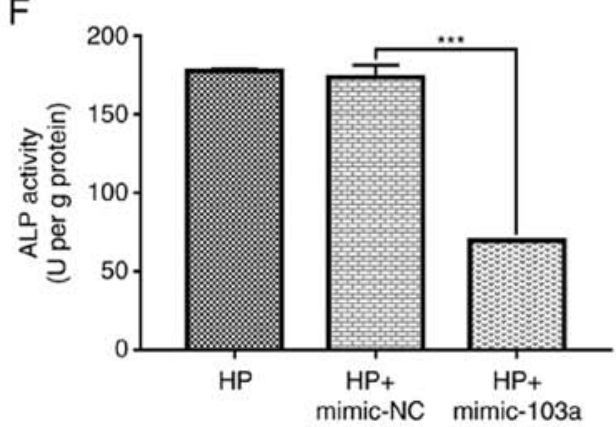

Figure 5. Effect of mimic-103a, inhibitor-103a or their respective NCs on VSMC phenotype. (A) Rat thoracic VSMCs treated with (a) normal medium, (b) normal medium with inhibitor NC or (c) normal medium with inhibitor miR-103a, for 3 days, followed by Alizarin Red staining; representative microscopic views are shown. Detection of (B) calcium content and (C) ALP activity after transfection with inhibitor miR-103a was quantified by spectrophotometry; both $\mathrm{n}=5$. (D) Rat thoracic VSMCs treated with (d) $10 \mathrm{mM} \beta$-GP, (e) $10 \mathrm{mM} \beta$-GP with mimic NC, or (f) normal medium with mimic miR-103a, for 3 days, followed by Alizarin Red S staining; representative microscopic views are shown. Detection of (E) calcium content and (F) ALP activity after transfection with mimic miR-103a was quantified by spectrophotometry; both $\mathrm{n}=5 .{ }^{* * * *} \mathrm{P}<0.001$. VSMCs, vascular smooth muscle cells; miR-, microRNA; ALP, alkaline phosphatase; NC, negative control; HP, high phosphorus; $\beta$-GP, $\beta$-glycerophosphate.

multiple regulatory elements (12). Therefore, the current study sought to identify potential miRNAs that bound the 3'-UTR of Runx2, and that suppressed its translation, as miRNAs have emerged as a novel regulatory mechanism for gene expression. Previous studies have shown that several miRNAs are involved in the modulation process of vascular calcification, such as miRNA-32 (21), miRNA-34 (13) and miRNA-204 (22). Based on the miRNA databases (http://www.mirbase.org/) and literature reports, seven miRNAs were selected (miR-29a, 30b, 103a, 125b, 133a, 143 and 211) to analyze the VSMC calcification model at 0,7 and 14 days. The miRNA databases predicted the sequence-specific base pairing of the 'seed region' at the 5'- or 3'- of miR-30b, -103a, -133a, -143 and -211 with the 3'-UTR binding sites of mRNA targets of Runx2 (Fig. 6); miR-29a and 125b were predicted from reports in the literature (23-25). Previous studies have shown that miR-29a, -30 , 


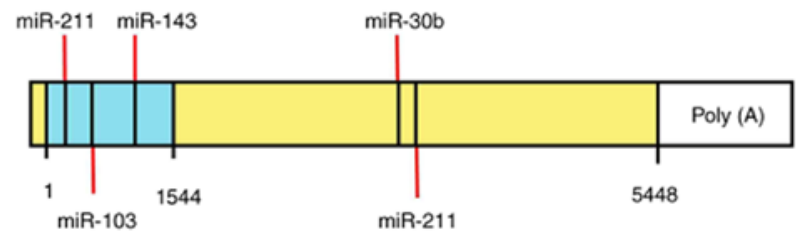

Hsa-miR-211 3' UCCGCUUCCUAC- UG- UUUCCCUU 5'
Runx2 464:5' CAGUUGAGG- UGCACUAAAGGGAC 3'

Hsa-miR-103 3' AGUAUCGGGACAUGUUACGACGA 5'

|| ||| | |||||||

Runx2 543:5' UUUCUGCUUUCAGACAUGCUGCA 3'

Hsa-miR-143 3' CUCGAUGUCACGAAGUAGAGU 5'
\[ |||| \mid \]

Runx2 1070:5' UUAAAAAAUACUUCCAUCUCC 3 '

Hsa-miR-30b 3' UCGACUCACAU - - CCUACAAAUGU 5'

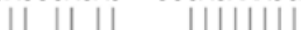

Runx2 3343:5' AUGUGUGUUUACUUCAUGUUUACA 3'

Hsa-miR-211 3' UCCGCUUCCUACUGUUUCCCUU 5

||||||||

Runx2 3639:5' UUACUCUUAACUUCAAAGGGAC 3'

Figure 6. Schematic of the microRNA-211, 103, 143 and 30b putative target sites in the Runx2 3' untranslated region, as determined by searching miRBase. miR-, microRNA; Runx2, runt-related transcription factor 2; has, homo sapiens; Poly(A), polyadenylated.

$-103 a,-125 b,-133 a,-143$ and -211 act as negative regulators of osteoblast differentiation and subsequent mineralization, functionally inhibiting the differentiation of osteoprogenitors by attenuating the essential transcription factor Runx2 (26-28).

The results of the present study revealed lower levels of miR-29a, 30b, 103a 125b and 143 in VSMCs following hyperphosphatemia, with an associated increase in Runx2 levels during the calcification process. However, miR-133a and miR-211 did not decrease significantly, although they did decrease in a similar manner to that reported previously (29). These results suggested that miR-133a and miR-211 may exert their effects on calcification through additional pathways (30).

In the present study, the expression of miR-103a was the most significantly reduced of the selected miRNAs in the VSMC calcification model. This finding indicated that miR-103a may be a powerful suppressor of VSMC calcification, and may have treatment potential for the process of vascular calcification. Therefore, miR-103a was the focus of further investigation herein. To determine how miR-103a regulated the osteo-/chondrogenic transdifferentiation of VSMCs, the effect of miR-103a on the expression of Runx 2 protein was determined. The results showed that miR-103a decreased the expression of Runx2 during the high phosphorus-induced phenotypic transition of VSMCs into osteoblast-like cells. Collectively, these findings indicate that miR-103a plays a notable role in the process of vascular calcification and ameliorates calcification by downregulating Runx2, which is consistent with the study by Zuo et al (31).

To further validate that miR-103 targeted the 3'-UTR of Runx2, Runx 2 wild-type and mutant derivative devoid of
miR-103-binding site were subcloned downstream of the coding region of the luciferase gene, and then used to transfect VSMCs. However, no significant downregulation in luciferase activity was observed following miR-103 overexpression in the present study.

A limitation of the current study was the lack of a positive finding of the direct link between miR-103 and Runx2. Moreover, the lack of investigation into the effects of high phosphorus and miR-103 on different Runx 2 isoforms is another limitation of this article. Therefore, in the future, further studies are required to investigate the targets of Runx2, to confirm the direct link between miR-103 and Runx2, and the effects of high phosphorus and miRNA-103 on different Runx 2 isoforms, for predicting or treating the high level of disease associated with vascular calcification.

In conclusion, the results of the current study showed that miR-103 alleviated calcification by inhibiting Runx 2 during high phosphorus-induced vascular calcification, which is a potential novel target for the treatment for this pathological phenomenon.

\section{Acknowledgements}

Not applicable.

\section{Funding}

The present study was funded by The Project of Hebei Clinical Medicine Outstanding Personnel Training (grant no. 2019139), The Hebei Province Medical Technology Tracking Project (grant no. G2018050) and Hebei province Key Research and Development Project (grant no. 20377704D).

\section{Availability of data and materials}

The datasets used and/or analyzed during the current study are available from the corresponding author on reasonable request.

\section{Authors' contributions}

JX and YB designed all experiments. $\mathrm{LH}, \mathrm{HZ}$ and $\mathrm{WZ}$ performed the experiments. MC, DZ, LZ and SZ analyzed the data. LH and SZ wrote the manuscript. JX and YB confirmed the authenticity of all the raw data. All authors have read and approved the final manuscript.

\section{Ethics approval and consent to participate}

All experimental programs were reviewed and approved by The Animal Care and Use Institutional Committee of Hebei Medical University (Shijiazhuang, China) (approval no. 2020ky189).

\section{Patient consent for publication}

Not applicable.

\section{Competing interests}

The authors declare that they have no competing interests. 


\section{References}

1. Kottgen A, Russell SD, Loehr LR, Crainiceanu CM Rosamond WD, Chang PP, Chambless LE and Coresh J: Reduced kidney function as a risk factor for incident heart failure: The atherosclerosis risk in communities (ARIC) study. J Am Soc Nephrol 18: 1307-1315, 2007.

2. Tentori F, Blayney MJ, Albert JM, Gillespie BW, Kerr PG, Bommer J, Young EW, Akizawa T, Akiba T, Pisoni RL, et al: Mortality risk for dialysis patients with different levels of serum calcium, phosphorus, and PTH: The Dialysis Outcomes and Practice Patterns Study (DOPPS). Am J Kidney Dis 52: 519-530, 2008.

3. Bolasco P: Effects of the use of non-calcium phosphate binders in the control and outcome of vascular calcifications: A review of clinical trials on CKD patients. Int J Nephrol 2011: 758450, 2011.

4. Adragao T: Evaluation of vascular calcifications in CKD patients. Int J Artif Organs 32: 81-86, 2009.

5. Bai Y, Zhang J, Xu J, Cui L, Zhang $\mathrm{H}$ and Zhang S: Alteration of type I collagen in the radial artery of patients with end-stage renal disease. Am J Med Sci 349: 292-297, 2015.

6. Towler DA and Demer LL: Thematic series on the pathobiology of vascular calcification: An introduction. Circ Res 108: 1378-1380, 2011.

7. Nitta $\mathrm{K}$ and Ogawa $\mathrm{T}$ : Vascular calcification in end-stage renal disease patients. Contrib Nephrol 185: 156-167, 2015.

8. Klimczak D, Paczek L, Jazdzewski K and Kuch M: MicroRNAs: Powerful regulators and potential diagnostic tools in cardiovascular disease. Kardiol Pol 73: 1-6, 2015.

9. Chiang VS: Withdrawn: MicroRNAs as potential regulators of docosahexaenoic acid benefits in Alzheimer's disease. Nutr Neurosci: Mar 14, 2015 (Epub ahead of print). doi: 10.1179/147 6830515Y.0000000014.

10. Mennigen JA, Plagnes-Juan E, Figueredo-Silva CA, Seiliez I, Panserat $S$ and Skiba-Cassy S: Acute endocrine and nutritional co-regulation of the hepatic omy-miRNA-122b and the lipogenic gene fas in rainbow trout, Oncorhynchus mykiss. Comp Biochem Physiol B Biochem Mol Biol 169: 16-24, 2014.

11. Pinto MT, Nicolete LD, Rodrigues ES, Palma PV, Orellana MD, Kashima S and Covas DT: Overexpression of hsa-miR-125b during osteoblastic differentiation does not influence levels of Runx2, osteopontin, and ALPL gene expression. Braz J Med Biol Res 46: 676-680, 2013.

12. Zhang W, Wu Y, Shiozaki Y, Sugimoto Y, Takigawa T, Tanaka M, Matsukawa A and Ozaki T: MiRNA-133a-5p inhibits the expression of osteoblast differentiation-associated markers by targeting the 3 UTR of RUNX2. DNA Cell Biol 37: 199-209, 2018.

13. Narayanan A, Srinaath N, Rohini M and Selvamurugan N: Regulation of Runx 2 by MicroRNAs in osteoblast differentiation. Life Sci 232: 116676, 2019.

14. Xu J, Bai Y, Jin J, Zhang J, Zhang S, Cui L and Zhang H: Magnesium modulates the expression levels of calcification-associated factors to inhibit calcification in a time-dependent manner. Exp Ther Med 9: 1028-1034, 2015.

15. Griffiths-Jones S, Grocock RJ, van Dongen S, Bateman A and Enright AJ: MiRBase: MicroRNA sequences, targets and gene nomenclature. Nucleic Acids Res 34: D140-D144, 2006.

16. Wang Z, Jiang Y, Liu N, Ren L, Zhu Y, An Y and Chen D: Advanced glycation end-product $\mathrm{N} \varepsilon$-carboxymethyl-Lysine accelerates progression of atherosclerotic calcification in diabetes. Atherosclerosis 221: 387-396, 2012.

17. Du Y, Wang Y, Wang L, Liu B, Tian Q, Liu CJ, Zhang T, Xu Q, Zhu Y, Ake O, et al: Cartilage oligomeric matrix protein inhibits vascular smooth muscle calcification by interacting with bone morphogenetic protein-2. Circ Res 108: 917-928, 2011.
18. Shanahan CM, Crouthamel MH, Kapustin A and Giachelli CM: Arterial calcification in chronic kidney disease: Key roles for calcium and phosphate. Circ Res 109: 697-711, 2011.

19. Tang Q, Tong M, Zheng G, Shen L, Shang P and Liu H Masquelet's induced membrane promotes the osteogenic differentiation of bone marrow mesenchymal stem cells by activating the Smad and MAPK pathways. Am J Transl Res 10: 1211-1219, 2018.

20. Luo Y, Cao X, Chen J, Gu J, Zhao J and Sun J: MicroRNA-224 suppresses osteoblast differentiation by inhibiting SMAD4. J Cell Physiol 233: 6929-6937, 2018.

21. Liu J, Xiao X, Shen Y, Chen L, Xu C, Zhao H, Wu Y, Zhang Q, Zhong J, Tang Z, et al: MicroRNA-32 promotes calcification in vascular smooth muscle cells: Implications as a novel marker for coronary artery calcification. PLoS One 12: e0174138, 2017.

22. Wang Y, Chen S, Deng C, Li F, Wang Y, Hu X, Shi F and Dong N: MicroRNA-204 Targets Runx2 to Attenuate BMP-2-induced osteoblast differentiation of human aortic valve interstitial cells. J Cardiovasc Pharmacol 66: 63-71, 2015.

23. Roberto VP, Tiago DM, Silva IA and Cancela ML: MiR-29a is an enhancer of mineral deposition in bone-derived systems. Arch Biochem Biophys 564: 173-183, 2014.

24. Wen P, Cao H, Fang L, Ye H, Zhou Y, Jiang L, Su W, Xu H, $\mathrm{He}$ W, Dai C and Yang J: MiR-125b/Ets1 axis regulates transdifferentiation and calcification of vascular smooth muscle cells in a high-phosphate environment. Exp Cell Res 322: 302-312, 2014.

25. Goettsch C, Rauner M, Pacyna N, Hempel U, Bornstein SR and Hofbauer LC: MiR-125b regulates calcification of vascular smooth muscle cells. Am J Pathol 179: 1594-1600, 2011.

26. Balderman JA, Lee HY, Mahoney CE, Handy DE, White K, Annis S, Lebeche D, Hajjar RJ, Loscalzo J and Leopold JA: Bone morphogenetic protein-2 decreases microRNA-30b and microRNA-30c to promote vascular smooth muscle cell calcification. J Am Heart Assoc 1: e003905, 2012.

27. Sun Z, Cao X, Hu Z, Zhang L, Wang H, Zhou H, Li D, Zhang S and Xie M: MiR-103 inhibits osteoblast proliferation mainly through suppressing Cav1.2 expression in simulated microgravity. Bone 76: 121-128, 2015.

28. Li E, Zhang J, Yuan T and Ma B: MiR-143 suppresses osteogenic differentiation by targeting Osterix. Mol Cell Biochem 390: 69-74, 2014.

29. Panizo S, Naves-Diaz M, Carrillo-Lopez N, Martinez-Arias L, Fernandez-Martin JL, Ruiz-Torres MP, Cannata-Andia JB and Rodriguez I: MicroRNAs 29b, 133b, and 211 regulate vascular smooth muscle calcification mediated by high phosphorus. J Am Soc Nephrol 27: 824-834, 2016.

30. Atlasi Y, Noori R, Gaspar C, Franken P, Sacchetti A, Rafati H, Mahmoudi T, Decraene C, Calin GA, Merrill BJ and Fodde R: Wnt signaling regulates the lineage differentiation potential of mouse embryonic stem cells through Tcf3 down-regulation. PLoS Genet 9: e1003424, 2013.

31. Zuo B, Zhu J, Li J, Wang C, Zhao X, Cai G, Li Z, Peng J, Wang P, Shen C, et al: MicroRNA-103a functions as a mechanosensitive microRNA to inhibit bone formation through targeting Runx2. J Bone Miner Res 30: 330-345, 2015.

This work is licensed under a Creative Commons Attribution-NonCommercial-NoDerivatives 4.0 International (CC BY-NC-ND 4.0) License. 